\title{
A New Hilbert-type integral inequality with a non-homogeneous kernel and its extension
}

\author{
Weiliang $\mathrm{Wu}^{1^{*}}$ \\ ${ }^{1}$ School of Mathematics Jiaying University, Guangdong Meizhou 514031, China \\ *Corresponding author E-mail:weilianggood@126.com
}

\begin{abstract}
By introducing some parameters, using the weight function and the technique of real analysis, a new Hilbert-type integral inequality with a non-homogeneous kernel as $\frac{1}{|1-a x y|^{\lambda_{2}}}(a \geq 1)$ and its equivalent form are established. As application, the constant factor on the plane is the best value and its extension form with some parameters is also considered.
\end{abstract}

Keywords: Some parameters, Hilbert-Type Integral Inequality, Best value, Extension.

\section{Introduction}

If $0<\int_{0}^{\infty} f^{2}(x) d x<\infty, 0<\int_{0}^{\infty} g^{2}(x) d x<\infty$, , Hilbert established the famous integral inequality ${ }^{[1]}$

$\int_{0}^{\infty} \int_{0}^{\infty} \frac{f(x) g(y)}{x+y} d x d y<\pi\left(\int_{0}^{\infty} f^{2}(x) d x \int_{0}^{\infty} g^{q}(y) d y\right)^{\frac{1}{2}}$,

where the constant factor $\pi$ is the best possible.(1) is important in analysis and its application ${ }^{[2-3]}$. Since 1998 , by introduced the conjugate index, a number of extensions (1) were given by Yang et al. ${ }^{[4-7]}$.

In this paper, by introducing some parameters and using the way of weight function, a new inequality with a non-homogeneous kernel as follows is established:

$\int_{0}^{\infty} \int_{0}^{\infty} \frac{1}{|1-a x y|^{1 / 2}}(a \geq 1) f(x) g(y) d x d y<$

$2 B\left(\frac{1}{2}, \frac{1}{4}\right)\left(\int_{0}^{\infty} x^{1 / 2} f^{2}(x) d x\right)^{\frac{1}{2}}\left(\int_{0}^{\infty} y^{1 / 2} g^{2}(y) d y\right)^{\frac{1}{2}}$.

where the constant factor $2 B\left(\frac{1}{2}, \frac{1}{4}\right)$ is the best possible. As application, the equivalent form and its extension form are obtained.

\section{Some Lemmas}

First, $\beta$ function is given ${ }^{[9]}$ :

$B(u, v)=\int_{0}^{1} x^{u-1}(1-x)^{v-1}=B(v, u)(u, v>0)$

Lemma 2.1. $0<\lambda_{1}<\lambda_{2}<1, a \geq 1, \eta\left(a, \lambda_{1}, \lambda_{2}\right):=$ $\frac{1}{a^{\lambda_{1}}}\left[B\left(\lambda_{1}, 1-\lambda_{2}\right)+B\left(\lambda_{2}-\lambda_{1}, 1-\lambda_{2}\right)\right], 0<\lambda_{1}<\lambda_{2}<1, a \geq$ $1, \eta\left(a, \lambda_{1}, \lambda_{2}\right):=\frac{1}{a^{\lambda_{1}}}\left[B\left(\lambda_{1}, 1-\lambda_{2}\right)+B\left(\lambda_{2}-\lambda_{1}, 1-\lambda_{2}\right)\right]$, define the following weight function:

$\omega(y):=\int_{0}^{\infty} \frac{1}{|1-a x y|^{\lambda_{2}}} \frac{y^{\lambda_{1}}}{x^{1-\lambda_{1}}} d y \quad(y>0)$, $\omega(x):=\int_{0}^{\infty} \frac{1}{|1-a x y|^{\lambda_{2}}} \frac{x^{\lambda_{1}}}{y^{1-\lambda_{1}}} d y \quad(x>0)$,

then we have

$\omega(x)=\omega(y)=\eta\left(a, \lambda_{1}, \lambda_{2}\right)$.

Proof Setting $u=$ axy and by (3),we obtain

$\omega(y)=\frac{1}{|1-u|^{\lambda_{2}}} \frac{y^{\lambda_{1}}}{\left(u y^{-1} a^{-1}\right)^{1-\lambda_{1}}} y^{-1} a^{-1} d u$

$$
\begin{aligned}
& =\frac{1}{a^{\lambda_{1}}}\left[\int_{0}^{1} \frac{1}{(1-u)^{\lambda_{2}}} u^{\lambda_{1}-1} d u+\int_{1}^{\infty} \frac{1}{(u-1)^{\lambda_{2}}} u^{\lambda_{1}-1} d u\right] \\
& =\frac{1}{a^{\lambda_{1}}}\left[B\left(\lambda_{1}, 1-\lambda_{2}\right)+B\left(\lambda_{2}-\lambda_{1}, 1-\lambda_{2}\right)\right]=\eta\left(a, v_{1}, v_{2}\right) .
\end{aligned}
$$

Similarly, we can calculate that $\omega(x)=\eta\left(a, v_{1}, v_{2}\right)$.

Lemma 2.2. As the assumption of Lemma 2.1, if $p>1, \frac{1}{p}+\frac{1}{q}=$ $1, f(x) \geq 0$, we have

$J:=\int_{0}^{\infty} y^{p \lambda_{1}-1}\left[\int_{0}^{\infty} \frac{1}{|1-a x y|^{\lambda_{2}}} f(x) d x\right]^{p} d y \leq$

$\left[\eta\left(a, \lambda_{1}, \lambda_{2}\right)\right]^{p} \int_{0}^{\infty} x^{p\left(1-\lambda_{1}\right)-1} f^{p}(x) d x$.

Proof By Hölder's inequality with weight ${ }^{[8]}$, we obtain $\int_{0}^{\infty} f(x) \frac{1}{|1-a x y|^{\lambda_{2}}} d x=\int_{0}^{\infty} \frac{1}{|1-a x y|^{\lambda_{2}}}\left[\frac{x^{\left(1-\lambda_{1}\right) / q}}{y^{\left(1-\lambda_{1}\right) / p}} f(x)\right]\left[\frac{y^{\left(1-\lambda_{1}\right) / p}}{x^{\left(1-\lambda_{1}\right) / q}}\right] d x$ $\leq\left\{\int_{0}^{\infty} \frac{1}{|1-a x y|^{\lambda_{2}}} \frac{x^{\left(1-\lambda_{1}\right)(p-1)}}{y^{\left(1-\lambda_{1}\right)}} f^{p}(x) d x\right\}^{1 / p}\left\{\int_{0}^{\infty} \frac{1}{|1-a x y|^{\lambda_{2}}} \frac{y^{\left(1-\lambda_{1}\right)(q-1)}}{x^{\left(1-\lambda_{1}\right)}} d x\right\}^{1 / q}$ $=y^{\frac{1}{p}-\lambda_{1}}[\omega(y)]^{1 / q}\left\{\int_{0}^{\infty} \frac{1}{|1-a x y|^{\lambda_{2}}} \frac{x^{\left(1-\lambda_{1}\right)(p-1)}}{y^{\left(1-\lambda_{1}\right)}} f^{p}(x) d x\right\}^{1 / p}$.

By (4)and Fubini's theorem ${ }^{[9]}$, we obtain

$\begin{aligned} J & \leq\left[\eta\left(a, \lambda_{1}, \lambda_{2}\right]^{p-1} \int_{0}^{\infty} \int_{0}^{\infty} \frac{1}{|1-a x y|^{\lambda_{2}}} \frac{x^{\left(1-\lambda_{1}\right)(p-1)}}{y^{\left(1-\lambda_{1}\right)}} f^{p}(x) d x d y\right. \\ & =\left[\eta\left(a, \lambda_{1}, \lambda_{2}\right]^{p-1} \int_{0}^{\infty} \omega(x) x^{p\left(1-\lambda_{1}\right)-1} f^{p}(x) d x .\right.\end{aligned}$ Hence by the above results, we have (5). The lemma is proved. 


\section{Main results}

Theorem 3.1. As the assumption of Lemma 2.1, if $p>$ $1, \frac{1}{p}+\frac{1}{q}=1, f(x), g(y) \geq 0,0<\int_{0}^{\infty} x^{p\left(1-\lambda_{1}\right)-1} f^{p}(x) d x<\infty, 0<$ $\int_{0}^{\infty} y^{q\left(1-\lambda_{1}\right)-1} g^{q}(y) d y<\infty$, then we have two equivalent inequalities as

$I:=\int_{0}^{\infty} \int_{0}^{\infty} \frac{1}{|1-a x y|^{\lambda_{2}}} f(x) g(y) d x d y<\eta\left(a, \lambda_{1}, \lambda_{2}\right) \times$

$\left\{\int_{0}^{\infty} x^{p\left(1-\lambda_{1}\right)-1} f^{p}(x) d x\right\}^{\frac{1}{p}}\left\{\int_{0}^{\infty} y^{q\left(1-\lambda_{1}\right)-1} g^{q}(y) d y\right\}^{\frac{1}{q}}$,

$J=\int_{0}^{\infty} y^{p \lambda_{1}-1}\left[\int_{0}^{\infty} \frac{1}{|1-a x y|^{\lambda_{2}}} f(x) d x\right]^{p} d y<$

$\left[\eta\left(a, \lambda_{1}, \lambda_{2}\right)\right]^{p} \int_{0}^{\infty} x^{p\left(1-\lambda_{1}\right)-1} f^{p}(x) d x$.

Proof By the conditions of theorem and Hölder's inequality,we conform that the middle of (6) keeps the form of strict inequality,Hence(6) takes the strict sign-inequality, so does (5). In addition, we have (7). By Hölder's inequality ${ }^{[8]}$, we find $I=\int_{0}^{\infty}\left[y^{\lambda_{1}-1 / p} \int_{0}^{\infty} \frac{1}{|1-a x y|^{\lambda_{2}}} f(x) d x\right]\left[y^{-\lambda_{1}+1 / p} g(y)\right] d y \leq$

$J^{1 / p}\left[\int_{0}^{\infty} y^{q\left(1-\lambda_{1}\right)-1} g^{q}(y) d y\right]^{1 / q}$.

Then by (8), we have (7).On the other hand, assuming that (7) is valid , setting $\left.g(y):=y^{p \lambda_{1}-1}\left[\int_{0}^{\infty} \frac{1}{|1-a x y|^{\lambda_{2}}} f(x)\right] d x\right]^{p-1}$

,then we have $J=\int_{0}^{\infty} y^{q\left(1-\lambda_{1}\right)-1} g^{q}(y) d y$, Through (5), it follows $J<$ $\infty$.IfJ $=0$, then (8)is naturally valid.If $0<J<\infty$, then by(7), we find $0<\int_{0}^{\infty} y^{q\left(1-\lambda_{1}\right)-1} g^{q}(y) d y=J=I<\eta\left(a, \lambda_{1}, \lambda_{2}\right) \times$

$\left\{\int_{0}^{\infty} x^{p\left(1-\lambda_{1}\right)-1} f^{p}(x) d x\right\}^{\frac{1}{p}}\left\{\int_{0}^{\infty} y^{q\left(1-\lambda_{1}\right)-1} g^{q}(y) d y\right\}^{\frac{1}{q}}$

$J^{1 / p}=\left\{\int_{0}^{\infty} y^{q\left(1-\lambda_{1}\right)-1} g^{q}(y) d y\right\}^{\frac{1}{p}}<$

$\eta\left(a, \lambda_{1}, \lambda_{2}\right)\left\{\int_{0}^{\infty} x^{p\left(1-\lambda_{1}\right)-1} f^{p}(x) d x\right\}^{\frac{1}{p}}$.

and then we have (8), which is equivalent to (7). The Theorem is proved.

Theorem 3.2. Under the conditions of Theorem 3.1, the constants $\eta\left(a, \lambda_{1}, \lambda_{2}\right)$ and $\left[\eta\left(a, \lambda_{1}, \lambda_{2}\right)\right]^{p}$ in (7) and (8) are the best value.

Proof For $0<\varepsilon<p v_{1}$, if

$\tilde{f}(x):=\left\{\begin{array}{cc}x^{\lambda_{1}+\varepsilon / p-1}, & x \in(0,1] \\ 0, & x \in(1, \infty)\end{array}\right.$

$\tilde{g}(y):=\left\{\begin{array}{cc}0, & y \in(0,1) \\ y^{\lambda_{1}-\varepsilon / q-1}, & y \in[1, \infty)\end{array}\right.$.

we can calculate

$\tilde{J}:=\left\{\int_{0}^{\infty} x^{p\left(1-\lambda_{1}\right)-1} \tilde{f}^{p}(x) d x\right\}^{\frac{1}{p}}\left\{\int_{0}^{\infty} y^{q\left(1-\lambda_{1}\right)-1} \tilde{g}^{q}(y) d y\right\}^{\frac{1}{q}}=\frac{1}{\varepsilon} \cdot b y$

Fubini's theorem ${ }^{[9]}$, we obtain

$I:=\int_{0}^{\infty} \int_{0}^{\infty} \frac{1}{|1-a x y|^{\lambda_{2}}} \tilde{f}(x) \tilde{g}(x) d x d y$

$=\int_{1}^{\infty} y^{\lambda_{1}-\varepsilon / q-1}\left[\int_{1}^{\infty} \frac{1}{|1-a x y|^{\lambda_{2}}} x^{\lambda_{1}+\varepsilon / p-1} d x\right] d y$

$(u=a x y) \frac{1}{a^{\lambda_{1}+\varepsilon / p}} \int_{1}^{\infty} y^{-\varepsilon-1}\left[\int_{0}^{a y} \frac{1}{|1-u|^{\lambda_{2}}} u^{\lambda_{1}+\varepsilon / p-1} d u\right] d y$

$=\frac{1}{a^{\lambda_{1}+\varepsilon / p}}\left\{\int_{1}^{\infty} y^{-\varepsilon-1}\left[\int_{0}^{1} \frac{u^{\lambda_{1}+\varepsilon / p-1}}{(1-u)^{\lambda_{2}}} d u+\int_{1}^{a y} \frac{u^{\lambda_{1}+\varepsilon / p-1}}{(u-1)^{\lambda_{2}}} d u\right] d y\right\}$

$=\frac{1}{a^{\lambda_{1}+\varepsilon / p}}\left\{\frac{1}{\varepsilon} \int_{0}^{1} \frac{u^{\lambda_{1}+\varepsilon / p-1}}{(1-u)^{\lambda_{2}}} d u+\int_{1}^{\infty}\left(\int_{a^{-1} u}^{\infty} y^{-\varepsilon-1}\right) \frac{u^{\lambda_{1}+\varepsilon / p-1}}{(u-1)^{\lambda_{2}}} d u\right\}$

$=\frac{1}{a^{\lambda_{1}+\varepsilon / p}}\left\{\frac{1}{\varepsilon} \int_{0}^{1} \frac{u^{\lambda_{1}+\varepsilon / p-1}}{(1-u)^{\lambda_{2}}} d u+\frac{a^{\varepsilon}}{\varepsilon} \int_{1}^{\infty} \frac{u^{\lambda_{1}-\varepsilon / q-1}}{(u-1)^{\lambda_{2}}} d u\right\}$.

If there exists a positive number $k \leq \eta\left(a, \lambda_{1}, \lambda_{2}\right)$,such that (7)is still valid when we replace $\eta\left(a, \lambda_{1}, \lambda_{2}\right)$ by $k$, then in particular $\tilde{f}, \tilde{g}$, by the above results, we find

$\frac{1}{a^{\lambda_{1}+\varepsilon / p}}\left\{\int_{0}^{1} \frac{u^{\lambda_{1}+\varepsilon / p-1}}{(1-u)^{\lambda_{2}}} d u+a^{\varepsilon} \int_{1}^{\infty} \frac{u^{\lambda_{1}-\varepsilon / q-1}}{(u-1)^{\lambda_{2}}} d u\right\}=$

$\varepsilon \tilde{I}<\varepsilon k \tilde{J}=k$.
By Fatou lemma ${ }^{[9]}$ and(12), we obtain $\eta\left(a, \lambda_{1}, \lambda_{2}\right)=$

$\frac{1}{a^{\lambda_{1}}}\left[\int_{0}^{1} \frac{\lim }{\varepsilon \rightarrow 0^{+}} \frac{1}{(1-u)^{\lambda_{2}}} u^{\lambda_{1}+\varepsilon / p-1} d u+\int_{1}^{\infty} \frac{\lim }{\varepsilon \rightarrow 0^{+}} \frac{1}{(1-u)^{\lambda_{2}}} u^{\lambda_{1}-\varepsilon / q-1} u\right] \leq$ $\frac{1}{a^{\lambda_{1}}} \underset{\varepsilon \rightarrow 0^{+}}{\lim }\left[\int_{0}^{1} \frac{1}{(1-u)^{\lambda_{2}}} u^{\lambda_{1}+\varepsilon / p-1} d u+\int_{1}^{\infty} \frac{1}{(u-1)^{\lambda_{2}}} u^{\lambda_{1}-\varepsilon / q-1} d u\right] \leq k$.

Hence $k=\eta\left(a, \lambda_{1}, \lambda_{2}\right)$ is the best value of (7). We conform that $\left[\eta\left(a, \lambda_{1}, \lambda_{2}\right)\right]^{p}$ in (8)is the best possible, otherwise we can get a contradiction by (9) that the constant in (7) is not the best possible.The theorem is proved.

\section{Conclusion}

For $a=1, \lambda_{1}=\frac{1}{4}, \lambda_{2}=\frac{1}{2}, p=q=2$ in (7), it deduces to (2). Hence inequality (7) is the best extension of (2).

\section{Acknowledgement}

The author would like to thank the editor and the referees. This work is supported by the key projects of Jiaying University(2014KJZ02) and College students' innovative training program of Guang$\operatorname{dong}(201510582021)$.

\section{References}

[1] Hardy GH.Note on a Theorem of H ilbert Concern ing Series of Positive Terms [J]. Proc London Math Soc,1925,23 (2):XLV-XLVL.

[2] Hardy GH,Littewood JE,PolyaG.Inequalities[M].Cambridge:Cambridge University Press, 1952

[3] Mitrinovic DS, Pecaric J, Fink AM. Inequalities Involving Functions and The ir Integrals and Derivatives [M ]. Boston:Kluwer Academic Publishers, 1991.

[4] Yongjing Li and Bing He. On inequalities of Hilbert's type [J].Bulletin of the Australian Mathematical Society,,2007,76(1):1-13.

[5] Qiong Liu. A Hilbert-type Integral Inequality with Several Parameters[J].J of Jinlin University:science edition,,2009,47(5):903-908.

[6] Bicheng Yang.On a Hilbert-type integral inequality with the homogeneous kernel of degree[J].J of Shanghai University(EnglEd):Natual Science, 2010,14(6):391-395.

[7] Weiliang Wu.A new Hilbert-type integral inequality with multiple independent parameters[J].J of Lanzhou university of technology,2015,41 (1):160-163.

[8] Jichang Kuang, Applied Inequalities, Shangdong Science Press, Jinan, 2004.

[9] Jichang Kuang, Introduction to real analysis, Hunan Education Press,Changsha,1996. 